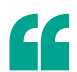

Gra15 is the first dense-granule protein found to alter host cell signalling

\title{
It takes II to induce NF-kB
}

Toxoplasma gondii is an obligate intracellular parasite that can infect a wide range of warm-blooded hosts by evading and subverting the immune response. There are three canonical strains of T. gondii, types I, II, and III, which vary greatly in virulence. Rosowski et al. now find a genetic basis for one important strainspecific difference in parasite-induced alteration of the host immune response: the high levels of host nuclear factor- $\kappa \mathrm{B}(\mathrm{NF}-\kappa \mathrm{B})$ activation after host cell infection.

To obtain a better insight into the interaction of the parasite with the host cell, Rosowski et al. focused on the differences between T. gondii strains. In particular,

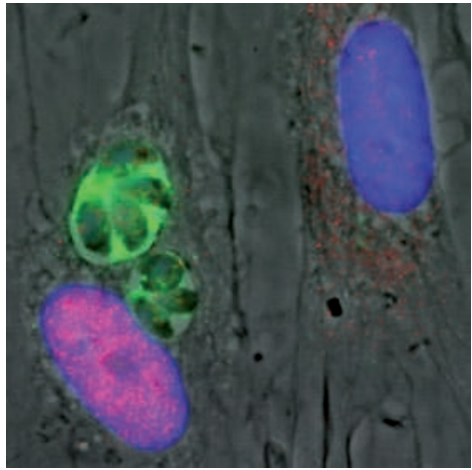

The human foreskin fibroblast on the left is infected with type I Toxoplasma gondii parasites expressing type II Gra15 (green). The nucleus of the infected cell shows clear nuclear translocation of the nuclear factor- $\kappa$ B p 65 subunit (pink). The cell on the right is not infected. Both nuclei are stained blue. Image courtesy of the Saeij Laboratory, Massachusetts Institute of Technology, Cambridge, USA they investigated the activation of the NF- $\kappa \mathrm{B}$ pathway, as previous reports had suggested that this pathway might mediate strain-specific differences in virulence. NF- $\kappa B$ activation is an important part of the immune response and leads to the secretion of cytokines such as the pro-inflammatory protein interleukin-12 (IL-12). When the authors infected human cells with each of the three types of T. gondii, they found that only the type II strain could significantly activate NF- $\kappa \mathrm{B}$, as measured by levels of nuclear translocation of the NF- $\kappa$ B subunit p 65

To identify the underlying genetic difference that lead to the differential activation of NF- $\kappa$ B, the authors used the $\mathrm{F}_{1}$ progeny of a cross between type II parasites (which activate NF- $\kappa \mathrm{B}$ ) and type III parasites (which do not) to map the locus responsible for the difference; the locus they identified encodes a previously unknown protein that they called Gra15. Gra15 encoded by type II parasites contains an 84 amino acid deletion - as well as several single amino acid substitutions - when compared with Gra15 in type I and type III parasites, possibly indicating how Gra15 from different strains can have different effects on the host cell. When the authors introduced type II Gra15 into type I and type III strains, they found that these transgenic strains could activate NF- $\kappa \mathrm{B}$, indicating that type II Gra15 is indeed responsible for the differences in activation of host NF- $\kappa B$. Furthermore, knocking out Gra15 in type II T. gondii decreases secretion levels of the NF- $\kappa B$ effector IL- 12 in mouse macrophages, and conversely, introducing type II Gra15 into type I T. gondii increases IL-12 secretion levels. Thus, type II Gra15 function is directly linked to the immune response.

To investigate the function of Gra15, the authors studied its localization and found that it is present in dense granules, which are specialized secretory organelles of $T$. gondii, and that the protein is secreted into the host cell during infection. Furthermore, the authors showed that activation of NF- $\kappa$ B by Gra 15 requires the host proteins inhibitor

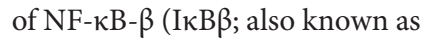
NF- $\kappa \mathrm{BIB}$ ) and TNF receptorassociated factor 6 (TRAF6). Gra15 is the first dense-granule protein found to alter host cell signalling, and it has a significant role in strainspecific modulation of host IL-12 expression. However, exactly how it functions - and how the strainspecific sequence differences relate to its function - remains to be fully understood.

Joanna E. Huddleston

ORIGINAL RESEARCH PAPER RosowskiE. E. etal. Strain-specific activation of the NF- $\mathrm{kB}$ pathway by Gra15, a novel Toxoplasma gondii dense granule protein J. Exp. Med. 208, 195-212 (2011) 